\title{
Improved correction of VIPERS angular selection effects in clustering measurements
}

\author{
A. Pezzotta ${ }^{1,2}$, B.R. Granett ${ }^{2}$, J. Bel ${ }^{2}$, L. Guzzo ${ }^{1,2}$, S. de la Torre ${ }^{3}$ \\ and VIPERS team* \\ ${ }^{1}$ Università di Milano Bicocca, Milano, Italy \\ ${ }^{2}$ INAF - Osservatorio Astronomico di Brera, Merate(LC), Italy \\ ${ }^{3}$ Aix, Marseille Université, CNRS, LAM, Marseille, France \\ email: pezzotta@brera.inaf.it \\ *see http://vipers.inaf.it for the full member list
}

\begin{abstract}
Clustering estimates in galaxy redshift surveys need to account and correct for the way targets are selected from the general population, as to avoid biasing the measured values of cosmological parameters. The VIMOS Public Extragalactic Redshift Survey (VIPERS) is no exception to this, involving slit collisions and masking effects. Pushed by the increasing precision of the measurements, e.g. of the growth rate $f$, we have been re-assessing these effects in detail. We present here an improved correction for the two-point correlation function, capable to recover the amplitude of the monopole of the two-point correlation function $\xi(r)$ above $1 \mathrm{~h}^{-1} \mathrm{Mpc}$ to better than $2 \%$.
\end{abstract}

Keywords. Large-scale structure, clustering, cosmological parameters

VIPERS has used the VIsible Multi-Object Spectrograph (VIMOS) at the ESO VLT to collect $\sim 90,000$ spectra for galaxies in the redshift range $0.5<z<1.2$. VIMOS spectra are collected within four distinct quadrants on the sky, corresponding to four metal masks on which slits are carved. With the VIPERS selection function and a single-pass strategy (see Guzzo et al. 2014), on average about $40 \%$ of the galaxies in the full parent sample get targeted by a slit. This fraction depends on the specific pointing/quadrant, defining a Target Sampling Rate $\left.\operatorname{TSR}_{i}(\mathrm{Q})\right)$. Simulations show that this introduces a constant $10 \%$ systematic damping in the amplitude of $\xi(r)$ on scales above $1 \mathrm{~h}^{-1} \mathrm{Mpc}$. This is essentially due to low-density regions being more represented than high-density ones in the overall pair counting. A second, interconnected effect happens at small angular separation due to "proximity bias", i.e. to the fact that two galaxies closer than a typical slit dimension cannot be both observed spectroscopically in a single visit. This effect, if not corrected, introduces a $50 \%$ systematic damping on scales below $1 \mathrm{~h}^{-1} \mathrm{Mpc}$.

To correct such effects, de la Torre et al.(2013) introduced appropriate weights when computing two-point correlations. Large-scale TSR variations were accounted for assigning to each galaxy a weight $w^{\mathrm{Q}}=1 / \mathrm{TSR}(\mathrm{Q})$. Then, each galaxy pair was then upweighted using an angular completeness function (Fig. 1), defined as the ratio between the angular correlation functions of the observed parent samples. With such correction, the original amplitude of the (projected) correlation function above $1 \mathrm{~h}^{-1} \mathrm{Mpc}$ could be recovered, for galaxies in the distant redshift bin of VIPERS $(z>0.75)$, but a residual lack of amplitude $\sim-4 \%$ remained at smaller redshifts. We present here an improvement over this early scheme.

To better account for the non-homogeneous sampling introduced by the target selection, we first introduce a galaxy up-weighting based on a more local value of the TSR. Rather than using a value averaged over a single quadrant, we define it over a smaller aperture of given shape and dimension around each galaxy. This is similar to what Pollo 

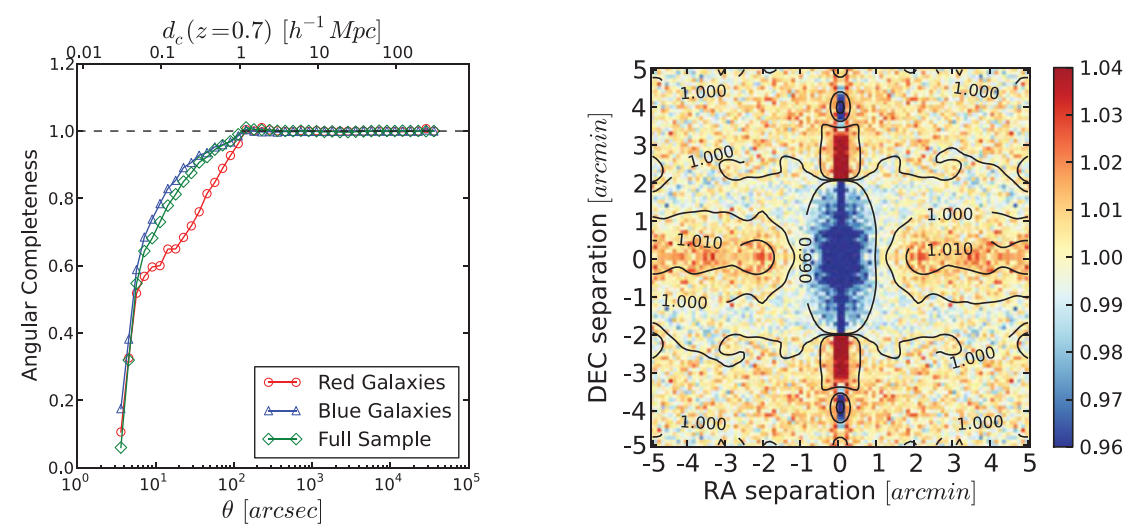

Figure 1.1d (left) and 2d (right) angular completeness functions computed using VIPERS mock samples. It is remarkable how the spectrum masking effect is able to reduce the completeness below a typical scale of about 100 arcsec.

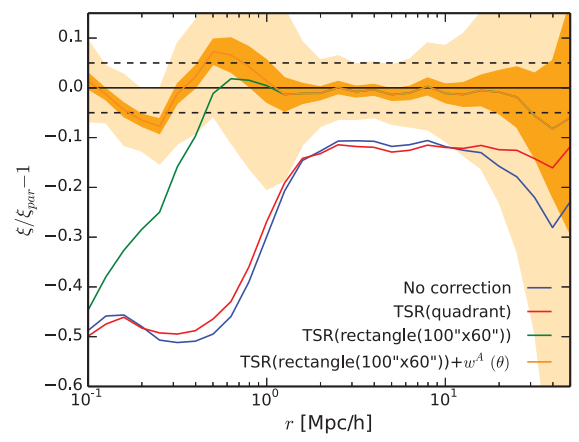

Figure 2. Fractional error on measurements of the real-space monopole $\xi(r)$. Shaded regions stand for the dispersion on the mean value (26 mocks) and on the single measurement for our final correction. $w^{A}$ marks the small-scale correction using the completeness function.

et al. (2005) developed for the VVDS-Deep survey. We test the impact of two different patterns, i.e. circular and rectangular. The latter shape is actually important as it mimicks the "shadow" of galaxy spectra, with its anisotropy between RA and DEC. The dimension of these regions are calibrated using the already cited angular completeness function (1d and $2 \mathrm{~d}$ respectively, Fig. 1). We couple this "local TSR" correction to the usual smaller-scale one based on the angular correlation functions. The results (Fig. 2) are excellent on a large set of statistical tools (projected correlation function, multipoles, power spectrum) and also for different colour classes and redshift bins. Specifically, we recover the clustering amplitude above $1 \mathrm{~h}^{-1} \mathrm{Mpc}$ to better than $\sim 2 \%$ using our improved TSR correction and to better than $5 \%$ below this scale, through the angular correlation function ratio.

\section{References}

Guzzo, L., the VIPERS team, 2014, A\&3 A, 566, A108

de la Torre, S., the VIPERS team, 2013, A\& A, 557, A54

Pollo, A., et al, 2005, A\&3 A, 439, 887-900 